\title{
Traces on multiplier Hopf algebras
}

\author{
L. Delvaux ${ }^{1}$
}

\section{Abstract}

In this article we lay the algebraic foundations to establish the existence of trace functions on infinite-dimensional (multiplier) Hopf algebras. We solve the problem within the framework of multiplier Hopf algebra with integrals. By applying this theory to group-cograded multiplier Hopf algebras, we prove the existence of group-traces on group-cograded multiplier Hopf algebras with possibly infinite-dimensional components. We generalize the results as obtained by A. Virelizier in the case of finite-type Hopf group-coalgebras.

\section{Introduction}

Quasitriangular ribbon Hopf algebras have a typical element with special properties in connection with the topology of knots and links. In the finite-dimensional case, the notion of a ribbon Hopf algebra is formulated in terms of grouplike elements, see e.g. [K-R]. Moreover, in the "unimodular" finite-dimensional case, the ribbon structure determines all traces which are invariant for the antipode, see $[\mathrm{H}]$.

In [Vir], the results are generalized to the framework of the so-called finite-type Hopf group-coalgebras. In this approach, one can consider an infinite-dimensional algebra, but all components have to be unital finite-dimensional. The proofs as given in [Vir] are based on known results for finite-dimensional Hopf algebras. In the present paper we generalize these algebraic structures to infinite-dimensional (multiplier) Hopf algebras. We solve the problem within the framework of multiplier Hopf algebras with integrals, the

\footnotetext{
${ }^{1}$ Department of Mathematics, Universiteit Hasselt, Agoralaan, B-3590 Diepenbeek, Belgium. E-mail: lydia.delvaux@uhasselt.be
} 
so-called algebraic quantum groups. These multiplier Hopf algebras are introduced and studied in [VD]. An important feature of this setting is the extension of the duality of finite-dimensional Hopf algebras, within one category. E.g. if $A$ is the group algebra of a group $G$, finite or not, then the dual exists in this framework and it is the multiplier Hopf algebra $K(G)$ of all functions with finite support and with the coproduct, properly defined, and dual to the product in $G$ (and the product in $A$ ). But this category of multiplier Hopf algebras also includes the duality between discrete quantum groups and compact quantum groups.

In the papers using multiplier Hopf algebras, we not only extend the results for finitedimensional Hopf algebras. On the contrary, we use different techniques. Essentially, we use integrals, both on the algebra and its dual. So it is not possible to have identities in both cases, except when each algebra is finite-dimensional. The use of non-unitial algebras is common in the field of operator algebra theory.

The underlying article is organised as follows. In Section 1 we work with multiplier Hopf algebras with integrals. We calculate the so-called modular functions, see Theorem 1.3. In the margin of these calculations, we recover Radford's formula for the fourth power of the antipode, see [R1]. The main consequence of Theorem 1.3 is given in Proposition 1.6. When we further restrict to the multiplier Hopf algebras of discrete type (which still contain all finite-dimensional Hopf algebras), we prove that all possible traces are included into the considered construction, see Proposition 1.9.

In Section 2 we realize multiplier Hopf algebras which satisfy the sufficient conditions as given in Proposition 1.6. We also allow a group-cograding on the multiplier Hopf algebra. The concept of a trace is adapted to this cograding, see Definition 2.1. However, when we take the group equal to the trivial group, we recover the usual concept of a trace which is commonly used in knot theory. By solving the problem into the group-cograding framework, our results can also be used in the sense of [Vir]. The main result is given in Theorem 2.6. When we restrict to the framework of discrete group-cograded multiplier Hopf algebras, the conditions of Theorem 2.6 can be reduced to only one condition, see 
Proposition 2.11. Recall that this class of multiplier Hopf algebras still contains the class of finite-type Hopf group-coalgebras.

In the Appendix we give an example of an infinite-dimensional discrete multiplier Hopf algebra which is ribbon quasitriangular. This multiplier Hopf algebra, denoted as $D_{q}\left(s l_{2}\right)$, is of interest because its multiplier algebra $M\left(D_{q}\left(s l_{2}\right)\right)$ contains the well-known quantum group $U_{q}\left(s l_{2}\right)$. Finally, we recall that the Drinfel'd double construction as obtained in [D-VD] leads to examples of group-cograded multiplier Hopf algebras with possibly infinite-dimensional components but which are still quasitriangular into the framework of group-cogradings.

\section{Notations and conventions}

Throughout the paper, we work with (associative) algebras over the complex numbers $\mathbb{C}$. We do not require the algebras to have an identity, but we do require that the product is non-degenerate (as a bilinear map). For an algebra $A$, we use $M(A)$ to denote the multiplier algebra. It is the largest unital algebra containing $A$ as a dense two-sided ideal. If both $A$ and $B$ are algebras and if $\alpha: A \rightarrow M(B)$ is an algebra homomorphism, then it is called non-degenerate if $\alpha(A) B=B$ and $B \alpha(A)=B$. In this case, $\alpha$ has a unique extension to a unital homomorphism from $M(A)$ to $M(B)$. This extension is still denoted by $\alpha$.

We use $1_{A}$ for the identidy in $M(A)$ and simply 1 if no confusion is possible. The identity element in a group is denoted by $e$. We use $\iota_{A}$ for the identity map from $A$ to itself and again, we simply write $\iota$ when appropriate. A multiplier Hopf algebra is an algebra $A$ with a coproduct $\Delta$ satisfying certain assumptions. The coproduct is a non-degenerate homomorphism from $A$ to $M(A \otimes A)$. It is assumed to be coassociative, i.e. we have $(\Delta \otimes \iota) \Delta=(\iota \otimes \Delta) \Delta$. A multiplier Hopf algebra is called regular if the opposite coproduct $\Delta^{o p}$ still makes $A$ into a multiplier Hopf algebra.

A regular multiplier Hopf algebra that carries integrals is called an algebraic quantum group. 


\section{Sufficient conditions for traces}

In knot theory, one usually considers finite-dimensional ribbon Hopf algebras, see e.g. [R2]. Virelizier has extended this point of view to finite-type Hopf group-coalgebras, see [Vir, Section7]. In this article, we construct traces on algebras which do not satisfy these finiteness conditions. We consider regular multiplier Hopf algebras with integrals, called algebraic quantum groups in [VD]. We recall the definition of the basic object. Observe that for any linear functional $\varphi$ on $A$ and any element $a \in A$, we can define the multiplier $(\iota \otimes \varphi) \Delta(a)$ in $M(A)$ by requiring

$$
\begin{aligned}
& ((\iota \otimes \varphi) \Delta(a)) x=(\iota \otimes \varphi)(\Delta(a)(x \otimes 1)) \text { and } \\
& x((\iota \otimes \varphi) \Delta(a))=(\iota \otimes \varphi)((x \otimes 1) \Delta(a)),
\end{aligned}
$$

for all $x \in A$. We use $\iota$ to denote the identity map on $A$. By the assumption that $(A, \Delta)$ is regular, we have that both $(x \otimes 1) \Delta(a)$ and $\Delta(a)(x \otimes 1)$ are in $A \otimes A$.

1.1 Definition [VD] A linear functional $\varphi$ on a regular multiplier Hopf algebra $A$ is called a left integral if $\varphi$ is non-zero and $(\iota \otimes \varphi) \Delta(a)=\varphi(a) 1$ in $M(A)$ for all $a \in A$. Similarly, a non-zero linear functional $\psi$ on $A$ satisfying $(\psi \otimes \iota) \Delta(a)=\psi(a) 1$ for all $a \in A$ is called a right integral on $A$.

In $[\mathrm{VD}]$, the existence of various relating data is shown. We need some of them. Let $\varphi$ be a left intergral. Any other left integral is a scalar multiple of $\varphi$. There is also a right integral $\psi$, unique up to a scalar, given by $\varphi \circ S$. There is a grouplike multiplier $\delta$ in $M(A)$ (the modular element) such that $(\varphi \otimes \iota) \Delta(a)=\varphi(a) \delta$ for all $a \in A$ and $(\iota \otimes \psi) \Delta(a)=\psi(a) \delta^{-1}$. The integrals are faithful in the sense that, for any $a \in A$, we have $a=0$ if either $\varphi(a b)=0$ for all $b \in A$ or $\varphi(b a)=0$ for all $b \in A$. Finally, there are automorphisms $\sigma$ and $\sigma^{\prime}$ (the modular automorphisms) satisfying $\varphi(a b)=\varphi(b \sigma(a)$ ) and $\psi(a b)=\psi\left(b \sigma^{\prime}(a)\right)$ for all $a, b \in A$. The modular automorphisms satisfy the relation $\delta \sigma(a)=\sigma^{\prime}(a) \delta$ for all $a \in A$. 
For a multiplier Hopf algebra $(A, \Delta)$ with a left integral $\varphi$, one has a natural notation of duality. Denote by $\widehat{A}$ the space of linear functionals on $A$ of the form, $x \rightarrow \varphi(x a)$ where $a \in A$. By the faithfulness of $\varphi$, the space $\widehat{A}$ separates the points of $A$ (that is, if $a, b \in A$ and $a \neq b$, then there is an element $f$ in $\widehat{A}$ such that $f(a) \neq f(b))$. Furthermore, one can show that $\widehat{A}$ is also the space of functionals of the form $x \rightarrow \varphi(a x)$, where $a$ runs through $A$. One can also use a right integral to define the space $\widehat{A}$. Then $\widehat{A}$ can be made into an algebra and there is a coproduct on $\widehat{A}$ making $(\widehat{A}, \widehat{\Delta})$ into a multiplier Hopf algebra with integrals. The product (resp. coproduct) of $\widehat{A}$ is dual to the coproduct (resp. product) of $A$. The dual of $(\widehat{A}, \widehat{\Delta})$ is canonically isomorphic with $(A, \Delta)$.

1.2 Lemma Let $A$ be a multiplier Hopf algebra with a left (resp. right) integral $\varphi(\operatorname{resp} . \psi)$. Let $\delta$ (resp. $\widehat{\delta})$ denote the modular element in $M(A)(\operatorname{resp} . M(\widehat{A}))$. Let $\alpha$ be an algebra automorphism of $A$ which respects the comultiplication in the sense that $\Delta(\alpha(a))=(\alpha \otimes \alpha) \Delta(a)$ for all $a \in A$. Under these conditions, $\alpha$ preserves the above data in the following way: $\varphi \circ \alpha=\lambda \varphi$ and $\psi \circ \alpha=\lambda \psi$ for some $\lambda \in \mathbb{C} \backslash\{0\}, \alpha(\delta)=\delta$ and $\widehat{\delta} \circ \alpha=\widehat{\delta}$.

Proof. It is easy to show that $\varepsilon(\alpha(a))=\varepsilon(a)$ and $S(\alpha(a))=\alpha(S(a))$ for all $a \in A$. We claim that $\varphi \circ \alpha$ is again a left integral on $A$. For all $a, b$ in $A$ we have

$$
\begin{aligned}
& ((\iota \otimes(\varphi \circ \alpha)) \Delta(a)) b=(\iota \otimes(\varphi \circ \alpha))(\Delta(a)(b \otimes 1)) \\
& =\sum(\varphi \circ \alpha)\left(a_{(2)}\right) a_{(1)} b=\sum \alpha^{-1}\left((\varphi \circ \alpha)\left(a_{(2)}\right) \alpha\left(a_{(1)} b\right)\right) \\
& =\alpha^{-1}(((\iota \otimes \varphi) \Delta(\alpha(a))) \alpha(b))=(\varphi \circ \alpha)(a) b .
\end{aligned}
$$

Therefore $(\iota \otimes(\varphi \circ \alpha)) \Delta(a)$ equals $(\varphi \circ \alpha)(a) 1$ as multipliers in $M(A)$.

Because integrals are unique, up to a scalar, $\varphi \circ \alpha=\lambda \varphi$ for some $\lambda \in \mathbb{C} \backslash\{0\}$. As $\psi=\varphi \circ S$, up to a scalar, the result for $\psi$ follows easily. Let $a$ be an element in $A$ such that $\varphi(a)=1$. By [VD, proposition 3.8], we have $\delta=(\varphi \otimes \iota) \Delta(a)$ in $M(A)$. This means that for all $b$ in $A$ we have $\delta b=\sum \varphi\left(a_{(1)}\right) a_{(2)} b$. So we obtain $\alpha(\delta) \alpha(b)=$ $\sum \varphi\left(a_{(1)}\right) \alpha\left(a_{(2)} b\right)=\lambda^{-1}((\varphi \otimes \iota) \Delta(\alpha(a))) \alpha(b)=\lambda^{-1} \varphi(\alpha(a)) \delta \alpha(b)=\delta \alpha(b)$. As $\alpha$ is an 
automorphism, we conclude that the multipliers $\delta$ and $\alpha(\delta)$ equal in $M(A)$. To obtain the result for the modular element in $M(\widehat{A})$, we observe that $\alpha$ defines an automorphism $\widehat{\alpha}$ on $\widehat{A}$ by the formula $\widehat{\alpha}(f)=f \circ \alpha$ for all $f \in \widehat{A}$. It is easy to see that $\widehat{\alpha}$ is an automorphism on $\widehat{A}$ which respects the comultiplication on $\widehat{A}$. Now the result for $\widehat{\delta}$ follows from the foregoing part in this proof.

We use the right $\widehat{A}$-module structure on $A$, denoted $A \triangleleft \widehat{A}$, and defined as follows. For $a, a^{\prime} \in A$, we define $a \triangleleft \varphi\left(a^{\prime} \cdot\right)=\sum \varphi\left(a^{\prime} a_{(1)}\right) a_{(2)}=(\varphi \otimes \iota)\left(\left(a^{\prime} \otimes 1\right) \Delta(a)\right)$ in $A$.

Observe that $A \triangleleft \widehat{A}$ defines an $\widehat{A}$-module structure on $A$ because the product in $\widehat{A}$ is dual to the coproduct in $A$. Analogously, we define the left action $\widehat{A} \triangleright A$ by the formula $\varphi\left(a^{\prime} \cdot\right) \triangleright a=\sum \varphi\left(a^{\prime} a_{(2)}\right) a_{(1)}=(\iota \otimes \varphi)\left(\left(1 \otimes a^{\prime}\right) \Delta(a)\right)$ in $A$. As $(A \otimes 1) \Delta(A)=A \otimes A=$ $(1 \otimes A) \Delta(A)$, we have that $\widehat{A} \triangleright A=A=A \triangleleft \widehat{A}$. Therefore, these actions extend in a natural way to the multiplier algebra $M(\widehat{A})$. Finally, we observe that $A$ is both a left and a right $\widehat{A}$-module algebra for these actions.

In general, integrals on multiplier Hopf algebras are not traces. However the formulas in Theorem 1.3 allow us to formulate conditions on $A$ in order to construct traces. In the margin of the first formulas, we obtain that Radford's formula for the fourth power of the antipode stays valid for this large class of multiplier Hopf algebras.

1.3 Theorem Let $A$ be a multiplier Hopf algebra with integrals. Let $\varphi$ (resp. $\psi$ ) denote a left (resp. right) integral on $A$. Let $\delta$ (resp. $\widehat{\delta}$ ) be the modular element in $M(A)$ (resp. $M(\widehat{A}))$. For all $x, y \in A$ we have

$$
\psi(x y)=\psi\left(y\left(S^{-2}(x) \triangleleft \widehat{\delta}^{-1}\right)\right) \quad \text { and } \quad \varphi(x y)=\varphi\left(y\left(\widehat{\delta}^{-1} \triangleright S^{2}(x)\right)\right) .
$$

Furthermore, $S^{4}(x)=\delta^{-1}\left(\widehat{\delta} \triangleright x \triangleleft \widehat{\delta}^{-1}\right) \delta$ for all $x \in A$.

Proof. The evaluation map of $\widehat{A}$ on $A$ is denoted as $\langle\widehat{A}, A\rangle$. This map can be extended to $\langle M(\widehat{A}), A\rangle$ as follows. For $m \in M(\widehat{A})$ and $a \in A$, we set $\langle m, a\rangle=\langle m b, a\rangle$ where 
$b$ is chosen in $\widehat{A}$ such that $a=b \boldsymbol{\Delta}$. It is not difficult to show that this definition of $\langle m, a\rangle$ does not depend on the element $b$. We claim that $\langle\widehat{\delta}, y\rangle=\varepsilon\left(\sigma^{-1}(y)\right)=\varepsilon\left(\sigma^{\prime}-1(y)\right)$ for all $y \in A$. To prove this claim, we choose any element $a \in A$ and we consider the element $\omega=\psi(a \cdot)$ in $\widehat{A}$. It is proven in [VD, Proposition 4.8] that a left integral $\widehat{\varphi}$ on $\widehat{A}$ is given by the formula $\widehat{\varphi}(\omega)=\varepsilon(a)$. For all $x$ and $y \in A$, we have

$$
\langle\omega, x y\rangle=\psi(a x y)=\psi\left(\sigma^{\prime-1}(y) a x\right)=\psi\left(\sigma^{\prime-1}(y) a \cdot\right)(x) .
$$

On the other hand, we have

$$
\langle\omega, x y\rangle=\langle\Delta(\omega), x \otimes y\rangle=\sum\left\langle\omega_{(1)}, x\right\rangle\left\langle\omega_{(2)}, y\right\rangle=\left(\sum\left\langle\omega_{(2)}, y\right\rangle \omega_{(1)}\right)(x) .
$$

As $\widehat{\widehat{A}} \cong A$, we conclude that $\psi\left(\sigma^{\prime}-1(y) a \cdot\right)=\sum\left\langle\omega_{(2)}, y\right\rangle \omega_{(1)}$.

By taking the left integral $\widehat{\varphi}$ on both sides of this equation, we obtain $\varepsilon\left(\sigma^{\prime}-1(y)\right) \varepsilon(a)=$ $\varepsilon(a)\langle\widehat{\delta}, y\rangle$ for all $a, y \in A$. Therefore we have $\langle\widehat{\delta}, y\rangle=\varepsilon\left(\sigma^{\prime}-1(y)\right)$ for all $y \in A$. Observe that $\delta \sigma(y)=\sigma^{\prime}(y) \delta$ for all $y \in A$ and $\sigma(\delta)=\lambda \delta$ for a non-zero scalar $\lambda$, see [VD, Proposition 3.15]. Therefore we also have $\langle\widehat{\delta}, y\rangle=\varepsilon\left(\sigma^{-1}(y)\right)$.

Next, we calculate $\widehat{\delta} \triangleright x$ for all $x \in A$. To ensure that all decompositions in $A$ are well-covered, we consider an arbitrary element $\omega=\varphi(a \cdot)$ in $\widehat{A}$. We have

$$
\langle\omega, \widehat{\delta}-x\rangle=\sum\left\langle\omega,\left\langle\widehat{\delta}, x_{(2)}\right\rangle x_{(1)}\right\rangle=\sum\left\langle\omega, \varepsilon\left(\sigma^{-1}\left(x_{(2)}\right)\right) x_{(1)}\right\rangle
$$

We observe that $\Delta(\sigma(x))=\left(S^{2} \otimes \sigma\right) \Delta(x)$ for all $x \in A$, see [VD, Proposition 3.14].

We now obtain that $\langle\omega, \widehat{\delta}-x\rangle=\left\langle\omega, S^{2}\left(\sigma^{-1}(x)\right)\right\rangle$ and therefore $\widehat{\delta} \bullet x=S^{2}\left(\sigma^{-1}(x)\right)$. This formula is equivalent to the identity $\sigma(x)=\widehat{\delta}^{-1} \triangleright S^{2}(x)$ for all $x \in A$.

By calculating the element $x \triangleleft \widehat{\delta}$, we obtain the automorphism $\sigma^{\prime}$. Indeed, let $\omega=\varphi(a \cdot)$ denote an arbitrary element in $\widehat{A}$. Then we have $\langle\omega, x \triangleleft \widehat{\delta}\rangle=\sum\left\langle\omega,\left\langle\widehat{\delta}, x_{(1)}\right\rangle x_{(2)}\right\rangle=$ $\sum\left\langle\omega, \varepsilon\left(\sigma^{\prime-1}\left(x_{(1)}\right)\right) x_{(2)}\right\rangle$.

We observe that $\Delta\left(\sigma^{\prime}(x)\right)=\left(\sigma^{\prime} \otimes S^{-2}\right) \Delta(x)$ for all $x \in A$, see [VD, Proposition 3.14].

Therefore we obtain that $\langle\omega, x \triangleleft \widehat{\delta}\rangle=\left\langle\omega, S^{-2}\left(\sigma^{\prime}-1(x)\right)\right\rangle$. By a similar reasoning as before, we obtain that $\sigma^{\prime}(x)=S^{-2}(x) \triangleleft \widehat{\delta}^{-1}$.

We have the equation $\delta \sigma(x)=\sigma^{\prime}(x) \delta$ for all $x \in A$, see [VD, Proposition 3.15]. By 
substituting the concrete realizations of $\sigma(x)$ and $\sigma^{\prime}(x)$ in this equation we obtain, for all $x \in A, S^{4}(x)=\delta^{-1}\left(\widehat{\delta} \triangleright x \triangleleft \widehat{\delta}^{-1}\right) \delta$.

1.4 Remark By the use of a new technique, Theorem 1.3 extends a result from finite-dimensional Hopf algebras to more general (multiplier) Hopf algebras.

1.5 Definition Let $A$ be a multiplier Hopf algebra with integrals. If the dual multiplier Hopf algebra $\widehat{A}$ is unimodular integral i.e. $\widehat{\delta}=1$ in $M(\widehat{A})$, then we call $A$ a counimodular multiplier Hopf algebra.

In classical Hopf algebra theory, a Hopf algebra $A$ such that $\widehat{\delta}=1$ is called "unimodular". However "counimodular" seems to us more appropriate, as it is consistent with the other Hopf terminology. Notice that in Knot theory it is very natural to assume counimodularity. This assumption is also motivated by the formulas in Theorem 1.3. Indeed, by the assumption that $\widehat{\delta}=1$, the square of the antipode takes care of the problem that the integrals are not traces.

1.6 Proposition Let $\psi$ (respectively $\varphi$ ) denote a right (respectively left) integral on a counimodular multiplier Hopf algebra $(A, \Delta)$. We have

(1) $\psi(x y)=\psi\left(y S^{-2}(x)\right)$ and $\varphi(x y)=\varphi\left(y S^{2}(x)\right)$ for all $x, y \in A$.

(2) If $m$ is a grouplike multiplier in $M(A)$, then we have $\varphi(\cdot m)=\varphi(m \cdot)$ and $\psi(\cdot m)=$ $\psi(m \cdot)$.

(3) For any multiplier $m$ in $M(A)$, the linear functional $\psi(m \cdot)$ is a trace on $A$ if and only if $S^{2}(a) m=m a$ for all $a \in A$. Similarly, we have that the linear functional $\varphi(\cdot m)$ is a trace on $A$ if and only if $m S^{2}(a)=a m$.

(4) Let $\delta$ denote the "modular element" in $M(A)$. For any multiplier $m \in M(A)$, we have $\psi(m S(\cdot))=\psi(m \cdot)$ if and only if $S(m)=m \delta$. 
For a left integral $\varphi$, we have $\varphi(S(\cdot) m)=\varphi(\cdot m)$ if and only if $S(m)=\delta^{-1} m$.

(5) Let $m$ be a grouplike multiplier in $M(A)$. Then $\psi(m \cdot)$ is a trace on $A$ so that $\psi(m S(a))=\psi(m a)$ for all $a$ in $A$ if and only if $S^{2}(a)=m a m^{-1}$ and $m^{2}=\delta^{-1}$. An analogue result yields for the left integral $\varphi$.

\section{Proof}

The statements (1)-(2)-(3) are straightforward calculations.

(4) We fix a multiplier $m \in M(A)$. We make use of [VD, Proposition 3.10] and Formula (1) of this proposition. We have, for all $a \in A, \psi(m S(a))=\psi\left(\delta^{-1} a S^{-1}(m)\right)=$ $\psi\left(a S^{-1}(m) \delta^{-1}\right)$. On the other hand, we also have $\psi(m a)=\psi\left(a S^{-2}(m)\right)$. As $\psi$ is faithful on $A$, we have that $\psi(m S(a))=\psi(m a)$ for all $a \in A$ if and only if $S^{-1}(m) \delta^{-1}=S^{-2}(m)$, or equivalently, $S(m)=m \delta$. The proof for the left integral $\varphi$ is similar.

(5) This statement follows from (3) and (4).

To finish this section, we restrict to the multiplier Hopf algebras of the so-called discrete type. A multiplier Hopf algebra is of discrete type if it has a cointegral. A left cointegral is a non-zero element $h$ in $A$ such that $a h=\varepsilon(a) h$ for all $a \in A$. One can prove that the existence of a left cointegral ensures there is also a right cointegral. Left and right cointegrals are unique up to a scalar, see [VD-Z, Proposition 2.7]. Multiplier Hopf algebras of discrete type share many nice properties with (the much smaller class of) finite-dimensional Hopf algebras, see [VD-Z]. In [VD, Proposition 5.3] is proven that all discrete multiplier Hopf algebras are duals of multiplier Hopf algebras of compact type and the other way around. A multiplier Hopf algebra is of compact type if it is an ordinary Hopf algebra with integrals (i.e. a co-Frobenius Hopf algebra). Lemma 1.7 expresses the crucial properties of the antipode in a multiplier Hopf algebra of discrete type. We use this lemma on several places in this paper. The evaluation map of $\widehat{A}$ on $A$ is denoted as $\langle\widehat{A}, A\rangle$. As before, we can extend this map to $\langle\widehat{A}, M(A)\rangle$. For a left integral $\varphi$ on $A$ and an element $a \in A$, consider $\varphi(a \cdot) \in \widehat{A}$. If $m \in M(A)$, we set $\langle\varphi(a \cdot), m\rangle=\varphi(a m) \in \mathbb{C}$. We recall that $A$ is a right $\widehat{A}$-module when the action is 
defined by the formula $a \triangleleft \varphi\left(a^{\prime} \cdot\right)=\sum \varphi\left(a^{\prime} a_{(1)}\right) a_{(2)}$ for all $a, a^{\prime} \in A$.

1.7 Lemma [D-VD-W] Let $h$ be a left cointegral in $A$. Let $\delta$ (resp. $\widehat{\delta}$ ) denote the modular element in $M(A)$ (resp. $M(\widehat{A})$ ). Then we have for all $a \in A$

(1) $h a=\langle\widehat{\delta}, a\rangle h$

(2) $(1 \otimes a) \Delta(h)=(S(a) \otimes 1) \Delta(h)$,

(3) $\Delta(h)(a \otimes 1)=\Delta(h)(1 \otimes S(a \triangleleft \widehat{\delta}))$ and

(4) $\Delta(h)(a \otimes 1)=\langle\widehat{\delta}, \delta\rangle^{-1} \sum S^{2}\left(h_{(2)}\right) a \otimes h_{(1)} \delta$.

1.8 Remark If $A$ is a classical Hopf algebra, cointegrals in $A$ are called "integrals in $A$ ". If $A$ is furthermore a finite-dimensional Hopf algebra, then there always exist both left and right cointegrals. They are, respectively, left and right integrals on the dual Hopf algebra $A^{\prime}$. Therefore, it seems to us more appropriate to use "cointegral" instead of "integral in" because it is consistent with the other Hopf terminology. From (1) in Lemma 1.7, we see that the modular element $\widehat{\delta}$ equals the "distinguished grouplike element" for finite-dimensional Hopf algebras.

1.9 Proposition Let $A$ be a counimodular discrete multiplier Hopf algebra. Let $\psi$ denote a right integral on $A$. A linear functional $f$ is a trace on $A$ if and only if $f=\psi(m \cdot)$, where $m$ is any multiplier in $M(A)$ satisfying the equation $S^{2}(a) m=m a$ for all $a \in A$.

Proof By Proposition 1.6(3), we only have to prove that each trace $f$ on $A$ takes the form $f=\psi(m \cdot)$, where $m$ is a multiplier in $M(A)$ satisfying the appropriate condition. Let $h$ be a cointegral in $A$. We define the multiplier $m$ in $M(A)$ by $m=(f \otimes \iota) \Delta(h)$. For all $a \in A$, we have

$$
\begin{aligned}
& a m=a((f \otimes \iota) \Delta(h))=(f \otimes \iota)((1 \otimes a) \Delta(h))=(f \otimes \iota)((S(a) \otimes 1) \Delta(h)) \\
& \left.=(f \otimes \iota)(\Delta(h)(S(a) \otimes 1))=(f \otimes \iota)\left(\Delta(h)\left(1 \otimes S^{2}(a)\right)\right)=(f \otimes \iota) \Delta(h)\right) S^{2}(a)=m S^{2}(a) .
\end{aligned}
$$


In the third step we use Lemma 1.7(2). In the fourth step we use the fact that $f$ is a trace and for the next step we use Lemma 1.7(3). Let $\varphi$ denote a left integral on $A$ and suppose that $\varphi(h)=1$. We see that the trace function $f \circ S$ equals the trace $\varphi(\cdot m)$, or equivalently $f=\psi(S(m) \cdot)$ where $\psi$ denotes the right integral $\psi=\varphi \circ S^{-1}$. Of course, we have $S^{2}(a) S(m)=S(m) a$ for all $a \in A$.

1.10 Remark In the Appendix we give a construction of infinite-dimensional counimodular discrete multiplier Hopf algebras which have a grouplike multiplier $m$, satisfying both conditions of Proposition 1.6(5).

\section{Construction of traces on multiplier Hopf algebras}

In this section we investigate whether the conditions in Proposition 1.6(5) are fulfilled. We solve this problem in a more general context. More precisely, we allow the multiplier Hopf algebra $A$ to be group-cograded by a group $G$, in the following sense.

(1) $A=\bigoplus_{p \in G} A_{p}$ with $\left\{A_{p}\right\}_{p \in G}$ a family of subalgebras such that $A_{p} A_{q}=0$ if $p \neq q$,

(2) $\Delta\left(A_{p q}\right)\left(1 \otimes A_{q}\right)=A_{p} \otimes A_{q}$ and $\left(A_{p} \otimes 1\right) \Delta\left(A_{p q}\right)=A_{p} \otimes A_{q}$ for all $p, q \in G$.

It is shown in [A-D-VD] that the data of a Hopf group-coalgebra, as introduced by Turaev in $[\mathrm{T}]$, give an example of a cograded multiplier Hopf algebra. By a crossing $\pi$ of the group $G$ on $A$, we mean a group homomorphism $\pi: G \rightarrow A u t(A)$. Further, for all $p \in G$ we require the following axioms.

(1) $\pi_{p}$ respects the comultiplication on $A$ in the sense that $\Delta\left(\pi_{p}(a)\right)=\left(\pi_{p} \otimes \pi_{p}\right)(\Delta(a))$ for all $a \in A$.

(2) $\pi_{p}\left(A_{q}\right)=A_{p q p^{-1}}$

Observe $M(A)=\prod_{p \in G} M\left(A_{p}\right)$. A multiplier in $M(A)$ is denoted by $m=\left(m_{p}\right)_{p \in G}$ where $m_{p} \in M\left(A_{p}\right)$. In this framework, we additionally require a trace to be invariant for the 
crossing $\pi$, in the sense of Definition 2.1(2). For a crossed Hopf-group-coalgebra, we recover the notion of a "group-trace" as defined in [Vir].

2.1 Definition Let $A$ be a $G$-cograded multiplier Hopf algebra and let $\pi$ be a crossing of $G$. A linear functional $f \in A^{\prime}$ is called a $\pi$-trace on $A$ if for all $x, y \in A$ the following conditions hold:

(1) $f(x y)=f(y x)$ and $f(S(x))=f(x)$,

(2) $f\left(\pi_{p}(x)\right)=f(x)$ for all $p \in G$.

When we set $G=\{e\}$, the results in this section can be used to construct traces on possibly infinite-dimensional (multiplier) Hopf algebras.

We assume that $A$ is counimodular in the sense of Definition 1.5. Let $\psi$ denote a right integral on $A$ and let $\delta$ denote the modular element in $M(A)$. For a grouplike multiplier $m$ in $M(A)$, we consider the linear functional $\psi(m \cdot)$. By Proposition 1.6(5) we have that $\psi(m \cdot)$ satisfies Condition (1) in Definition 2.1 if and only if $S^{2}(x)=m x m^{-1}$ for all $x \in A$ and $m^{2}=\delta^{-1}$. To find a grouplike multiplier $m$ in $M(A)$ which satisfies both these conditions we require that $A$ is $\pi$-quasitriangular in the sense of [D-VD-W].

We briefly recall the fundamental definitions of the basic ideas which are used in [DVD-W]. First, we have to consider a deformation $\widetilde{A}$ of $A$ in the following way. As algebras, we set $\widetilde{A}=A$. The comultiplication $\widetilde{\Delta}$ on $\widetilde{A}$ is defined by $\widetilde{\Delta}(a)\left(1 \otimes a^{\prime}\right)=$ $\left(\pi_{q-1} \otimes \iota\right)\left(\Delta(a)\left(1 \otimes a^{\prime}\right)\right)$, where $a \in A$ and $a^{\prime} \in A_{q}$. The counit $\widetilde{\varepsilon}$ is the original counit $\varepsilon$. The antipode $\widetilde{S}$ is given by the formula $\widetilde{S}(a)=\pi_{p-1}(S(a))$ for all $a \in A_{p}$. One can show that $\widetilde{A}$ is a regular multiplier Hopf algebra.

We call $A \pi$-quasitriangular if there is an invertible multiplier $R \in M(A \otimes A)$ such that

(1) $\left(\pi_{p} \otimes \pi_{p}\right)(R)=R$ for all $p \in G$,

(2) $R \Delta(a)=\widetilde{\Delta}^{o p}(a) R$ for all $a \in A$, 
$(3)(\widetilde{\Delta} \otimes \iota)(R)=R^{13} R^{23}$ and

(4) $(\iota \otimes \Delta)(R)=R^{13} R^{12}$.

We call $R$ the generalized $\pi$-matrix for $A$. For all $a \in A$, we require that both $R(a \otimes 1)$ and $(a \otimes 1) R$ sit in $A \otimes M(A)$. We denote the element $R(a \otimes 1)$ in $A \otimes M(A)$ by $\sum R^{(1)} a \otimes R^{(2)}$. A similar notation is used for $(a \otimes 1) R$. The $\pi$-Drinfel'd multiplier $u$ is an invertible multiplier in $M(A)$. As a left multiplier, we have $u a=\sum \widetilde{S}\left(R^{(2)}\right) R^{(1)} a$ for all $a \in A$. As a right multiplier, we have $a u=u \pi_{p-1}\left(S^{-2}(a)\right)$ for all $a \in A$. The square of the antipode is given by the formula $S^{2}(a)=u\left(\pi_{p-1}(a)\right) u^{-1}$ for all $a \in A_{p}$. Further, we impose extra conditions on $A$ in the sense of the following definition.

2.2 Definition Let $A$ be a $G$-cograded multiplier Hopf algebra which is $\pi$-quasitriangular for a given crossing $\pi$ of $G$ and let $R$ denote the " $\pi$-matrix" in $M(A \otimes A)$. We call $A$ a $\pi$-ribbon multiplier Hopf algebra if there is a invertible multiplier $\theta \in M(A)$ such that, for all $p, q \in G$,

(1) $\pi_{p}(\theta)=\theta$,

(2) $S(\theta)=\theta$,

(3) $\pi_{p}(a)=\theta^{-1} a \theta$ for $a \in A_{p}$ and

(4) $\Delta(\theta)\left(1_{p} \otimes 1_{q}\right)=(\theta \otimes \theta)\left(\left(\iota \otimes \pi_{p^{-1}}\right)(\sigma(R))\right) R\left(1_{p} \otimes 1_{q}\right)$,

where $\sigma$ denotes the usual flip map, extended to $M(A \otimes A)$.

2.3 Remarks (1) Write $A=\bigoplus_{p \in G} A_{p}$. If each algebra $A_{p}$ has a unit element, then $A$ is a Hopf $G$-coalgebra in the sense of $[\mathrm{T}]$. In this case we recover the definition of a ribbon Hopf group-coalgebra as given in [T, Section 14].

(2) In Definition 2.2 we make use of the extension of a non-degenerate homomorphism to the multiplier algebra. We have $A=\bigoplus_{p \in G} A_{p}$ and so $M(A)=\prod_{p \in G} M\left(A_{p}\right)$. The multiplier $\theta \in M(A)$ can be written as $\theta=\left(\theta_{p}\right)_{p \in G}$ with $\theta_{p} \in M\left(A_{p}\right)$.

(3) Let $w \in M(A)$ and $\pi_{p}(w)=w$ for all $p \in G$. Then we have $w \theta=\theta w$. 
(4) Put $G=\{e\}$. In the case where $A$ is a usual Hopf algebra we recover the definition of a ribbon Hopf algebra as given in [R2, Section 2.2]. Remark that Radford's ribbon element is the inverse of the ribbon element $\theta$ as considered in Definition 2.2.

2.4 Proposition Let $A$ be a $\pi$-ribbon multiplier Hopf algebra in the sense of Definition 2.2 and let $u$ denote the $\pi$-Drinfel'd multiplier of the $\pi$-matrix $R$. Define $m$ in $M(A)$ as $m=\theta u$. We have

(1) $m=u \theta$ and $m$ is a grouplike multiplier in $M(A)$,

(2) $S^{2}(a)=\operatorname{mam}^{-1}$, for all $a \in A$, and

(3) $m^{2}=u S(u)^{-1}=S(u)^{-1} u$.

Proof (1) For all $p \in G$ we have $\pi_{p}(u)=u$. By Remark 2.3(3), $u$ commutes with $\theta$. By [D-VD-W, Lemma 3.10], we have for all $p, q \in G$

$$
\Delta(u)\left(1_{p} \otimes 1_{q}\right)=R^{-1}\left(\left(\iota \otimes \pi_{p^{-1}}\right)\left(\sigma\left(R^{-1}\right)\right)\right)(u \otimes u)\left(1_{p} \otimes 1_{q}\right) .
$$

By the formula above, we easily see that $m=\theta u$ is a grouplike multiplier.

(2) If $p \in G$ and $a \in A_{p}$, then we have $S^{2}(a)=u \pi_{p^{-1}}(a) u^{-1}$. By (3) in Definition 2.2, we obtain $S^{2}(a)=u \theta a \theta^{-1} u^{-1}=\operatorname{mam}^{-1}$.

(3) We observe that $\left(\theta^{-1}\right)^{2}=u S(m) \theta^{-1}=u S(\theta u) \theta^{-1}=u S(u) S(\theta) \theta^{-1}=u S(u)$.

By [D-VD-W, Lemma 3.7], we have $\pi_{p}(a) u S(u)=u S(u) \pi_{p-1}(a)$ for all $p \in G$ and $a \in A_{p}$. As $\pi_{p}(u)=u$ for all $p \in G$, we obtain $u S(u)=S(u) u$. Finally we have $u S(u)^{-1}=u^{2} \theta^{2}=(u \theta)^{2}=m^{2}$.

The following definition is motivated by Lemma 1.2.

2.5 Definition Let $A=\bigoplus_{p \in G} A_{p}$ denote a $G$-cograded multiplier Hopf algebra and let $\pi$ denote a crossing of $G$ on $A$. Suppose that $\varphi$ (resp. $\psi$ ) is a left (resp. right) integral on $A$. For all $p \in G$, we define the crossing scaling as the scalar $\rho_{p}$ such that $\varphi \circ \pi_{p}=\rho_{p} \varphi$. 
Remark that we also have $\psi \circ \pi_{p}=\rho_{p} \psi$ for all $p \in G$.

2.6 Theorem Let $A$ be a multiplier Hopf algebra with integrals and assume $A$ to be counimodular. Furthermore suppose $A$ to be $G$-cograded and let $\pi$ be a crossing of $G$ such that $A$ is $\pi$-ribbon. Let $m$ denote the grouplike multiplier $m=\theta u$ where $u$ is the $\pi$-Drinfel'd multiplier and $\theta$ is the $\pi$-ribbon multiplier. Let $\psi$ denote a right integral on $A$ and let $\delta$ denote the modular element in $M(A)$. The linear functional $\psi(m \cdot)$ is a $\pi$-trace on $A$ if and only if (1) $u S(u)^{-1}=\delta^{-1}$ and (2) $\rho_{p}=1$ for all $p \in G$.

Proof First, we combine Proposition 1.6(5) and Proposition 2.4(2)-(3) . Next, we observe that for all $p \in G$ and for all $a \in A, \psi\left(m \pi_{p}(a)\right)=\psi\left(\pi_{p}(m a)\right)=\rho_{p} \psi(m a)$. We obtain that $\psi\left(m\left(\pi_{p}(a)\right)\right)=\psi(m a)$ for all $a \in A$ if and only if $\rho_{p}=1$ for all $p \in G$.

In Proposition 2.7 we give situations where the condition $\rho_{p}=1$, for all $p \in G$, is fulfilled.

2.7 Proposition Let $A$ be a multiplier Hopf algebra as in Theorem 2.6. Let $\psi_{p}$ denote the restriction of $\psi$ to the subalgebra $A_{p}$. In each of the situations (1)-(2), we have $\rho_{p}=1$ for all $p \in G$.

(1) There exists an element $a \in A_{e}$, such that $\psi_{e}(a) \neq 0$ and $\pi_{p}(a)=a$ for all $p \in G$.

(2) For all $p \in G$, there exists an element $a_{p} \in A_{p}$ such that $\psi_{p}\left(a_{p}\right) \neq 0$ and $\pi_{p}\left(a_{p}\right)=a_{p}$.

\section{Proof}

(1) For all $p \in G$, we have $\psi_{e} \circ \pi_{p}=\rho_{p} \psi_{e}$. Let $a$ be in $A_{e}$ and suppose $\pi_{p}(a)=a$, then we have $\psi_{e}(a)=\rho_{p} \psi_{e}(a)$ and the result follows.

(2) For all $p \in G$, we have $\psi_{p} \circ \pi_{p}=\rho_{p} \psi_{p}$. If $a_{p} \in A_{p}$ is such that $\pi_{p}\left(a_{p}\right)=a_{p}$ then we have $\psi_{p}\left(a_{p}\right)=\rho_{p} \psi_{p}\left(a_{p}\right)$ and $\rho_{p}=1$ when $\psi_{p}\left(a_{p}\right) \neq 0$. Suppose that for all $p \in G$ the algebra $A_{p}$ is unital. Then the element $a_{p}$ can be taken as $a_{p}=1_{p}$ when $\psi_{p}\left(1_{p}\right) \neq 0$. 
In the sequel, we restrict to the category of discrete multiplier Hopf algebras and we sharp the result of Theorem 2.6. Recall from [VD, Proposition 5.3] that the discrete multiplier Hopf algebras are the duals (in the sense of [VD]) of the co-Frobenius Hopf algebras (i.e. Hopf algebras with integrals). So, if $A$ is a discrete multiplier Hopf algebra, then we have $M(\widehat{A})=\widehat{A}$.

2.8 Lemma Let $A$ be a discrete $G$-cograded multiplier Hopf algebra and put $A=\bigoplus_{p \in G} A_{p}$. Then each cointegral is in $A_{e}$. For the modular element $\widehat{\delta}$ in $\widehat{A}$, we have $\widehat{\delta} \in \widehat{A}_{e}$.

Proof. For the counit $\varepsilon$ we have $\varepsilon\left(A_{p}\right)=0$ when $p \neq e$. Let $h$ be a left cointegral in $A$. For $p \in G$ and $a \in A_{p}$, we have that $a h=0$ whenever $p \neq e$. As each subalgebra $A_{p}$ has a non-degenerate multiplication, we easily obtain that $h \in A_{e}$. The proof for a right cointegral is similar. By Lemma 1.7(1) and the first part of this proof, we easily see that $\widehat{\delta} \in \widehat{A}_{e}$.

We now generalize the result of [Vir, Theorem 6.9] on finite-type quasitriangular Hopf group-coalgebras. Suppose $A$ is a discrete $G$-cograded multiplier Hopf algebra. Put $A=\bigoplus_{p \in G} A_{p}$. By Lemma 2.8 we have that $A_{e}$ is a discrete multiplier Hopf algebra. Let $\pi$ be a crossing of $G$. Suppose $A$ is $\pi$-quasitriangular. Let $u$ denote the $\pi$-Drinfel'd multiplier in $M(A)$ according to a $\pi$-R-matrix. We give a relation between the modular element $\delta$ in $M(A)$ and the grouplike multiplier $u S(u)^{-1}$.

2.9 Theorem Let $A$ be a discrete $G$-cograded multiplier Hopf algebra, $A=\bigoplus_{p \in G} A_{p}$. Let $\delta=\left(\delta_{p}\right)_{p \in G}$ denote the modular element in $M(A)$. Let $\pi$ denote a crossing of $G$ and suppose $A$ is $\pi$-quasitriangular. The modular element $\delta$ is determined by the Drinfel'd 
multiplier $u$ in the following way:

$$
\delta_{p}^{-1}=\rho_{p^{-1}} u S(u)^{-1}(\iota \otimes\langle\widehat{\delta}, \cdot\rangle)(R) 1_{p}
$$

for all $p \in G$.

Proof. By applying Lemma 2.8, we have that $A_{e}$ is a multiplier Hopf algebra of discrete type and so $M\left(A_{e}\right)=\left(\widehat{A}_{e}\right)^{\prime}$, see [VD-Z, Proposition 2.9]. Observe that we first take the dual in the sense of $[\mathrm{VD}]$ and then the classical linear dual. Therefore, $(\iota \otimes\langle\widehat{\delta}, \cdot\rangle)(R)$ is a multiplier in $M(A)$ in the following way. For $a \in A$, we have $((\iota \otimes\langle\widehat{\delta}, \cdot\rangle)(R)) a=$ $\sum\left\langle\widehat{\delta}, R^{(2)}\right\rangle R^{(1)} a \in A$ because $R(a \otimes 1)$ is in $A \otimes M(A)$. Similarly, we obtain that $(\iota \otimes\langle\widehat{\delta}, \cdot\rangle)(R)$ is a right multiplier of $A$. By the associativity of the product in $A$, we have that $(\iota \otimes\langle\widehat{\delta}, \cdot\rangle)(R)$ is a multiplier in $M(A)$.

In the proof we take the notation $R(a \otimes 1)=\sum R^{(1)} a \otimes R^{(2)}=\sum r^{(1)} a \otimes r^{(2)}$ in $A \otimes M(A)$. We use the deformed multiplier Hopf algebra $\widetilde{A}$ as defined before. Let $h$ be a left cointegral in $A_{e}$. For all $p \in G$ and $a, a^{\prime} \in A_{p}$, we have in $A \otimes A$

$$
(a \otimes 1) R \Delta(h)\left(a^{\prime} \otimes 1\right) \stackrel{(*)}{=}(a \otimes 1) \widetilde{\Delta}^{c o p}(h) R\left(a^{\prime} \otimes 1\right)
$$

By Lemma 1.7, we rewrite the left hand side of equation $(*)$ as

$$
\begin{aligned}
& (a \otimes 1) R \Delta(h)\left(a^{\prime} \otimes 1\right)=\sum a R^{(1)} h_{(1)} a^{\prime} \otimes R^{(2)} h_{(2)}=\sum a R^{(1)} S\left(R^{(2)}\right) h_{(1)} a^{\prime} \otimes h_{(2)} \\
& =\sum a S(u) h_{(1)} a^{\prime} \otimes h_{(2)}=\langle\widehat{\delta}, \delta\rangle^{-1} \sum a S(u) S^{2}\left(h_{(2)}\right) a^{\prime} \otimes h_{(1)} \delta=\sum a S(u) S^{2}\left(h_{(2)}\right) \delta^{-1} a^{\prime} \otimes h_{(1)} .
\end{aligned}
$$

Again, by Lemma 1.7, we rewrite the right hand side of equation $(*)$ as

$$
\begin{aligned}
(a \otimes 1) \widetilde{\Delta}^{c o p}(h) R\left(a^{\prime} \otimes 1\right) & =\sum a h_{(2)} R^{(1)} a^{\prime} \otimes \pi_{p^{-1}}\left(h_{(1)} \pi_{p}\left(R^{(2)}\right)\right) \\
& =\sum a h_{(2)} S\left(\pi_{p}\left(R^{(2)}\right) \triangleleft \widehat{\delta}\right) R^{(1)} a^{\prime} \otimes \pi_{p^{-1}}\left(h_{(1)}\right) .
\end{aligned}
$$

In the expression above we use the action $A \triangleleft \widehat{A}$, as defined in Section 1 and extended to $M(A) \triangleleft \widehat{A}$. By the use of the properties of the $\pi$-quasitriangular $R$-matrix and the use of Lemma 1.2, we find successively the following expressions for the right hand side 
of equation $(*)$

$$
\begin{aligned}
\sum\left\langle\widehat{\delta}, \pi_{p}\left(r^{(2)}\right)\right\rangle a h_{(2)} S\left(\pi_{p}\left(R^{(2)}\right)\right) R^{(1)} r^{(1)} a^{\prime} \otimes \pi_{p^{-1}}\left(h_{(1)}\right) & \\
=\sum\left\langle\widehat{\delta}, \pi_{p}\left(r^{(2)}\right)\right\rangle & a h_{(2)} u r^{(1)} a^{\prime} \otimes \pi_{p^{-1}}\left(h_{(1)}\right) \\
& =\sum\left\langle\widehat{\delta}, r^{(2)}\right\rangle a h_{(2)} u r^{(1)} a^{\prime} \otimes \pi_{p^{-1}}\left(h_{(1)}\right) .
\end{aligned}
$$

For all $p \in G$ and $a \in A_{p}$ we have obtained that the equation $(*)$ is equivalent to

$$
\sum a S(u) S^{2}\left(h_{(2)}\right) \delta^{-1} a^{\prime} \otimes h_{(1)}=\sum\left\langle\widehat{\delta}, r^{(2)}\right\rangle a h_{(2)} u r^{(1)} a^{\prime} \otimes \pi_{p^{-1}}\left(h_{(1)}\right) .
$$

For any right integral $\psi$, we have $\psi(h) \neq 0$. So we can assume $\psi(h)=1$. By applying the operator $(\iota \otimes \psi)$ on both sides of the above equation, we obtain for all $p \in G$ and $a \in A_{p}$

$$
S(u) \delta^{-1} a=\rho_{p^{-1}} \sum\left\langle\widehat{\delta}, r^{(2)}\right\rangle u r^{(1)} a=\rho_{p^{-1}} u((\iota \otimes\langle\widehat{\delta}, \cdot\rangle)(R)) a .
$$

This equation is equivalent to the equation in the statement of the theorem.

2.10 Corollaries Let $A$ be a discrete multiplier Hopf algebra as in Theorem 2.9.

(1) In Theorem 1.3, we have proven Radford's formula for the fourth power of the antipode. As $A$ is a $\pi$-quasitriangular multiplier Hopf algebra, we also have $S^{4}(x)=$ $u S(u)^{-1} x S(u) u^{-1}$ for all $x \in A$. The formula in Theorem 2.9 gives a connection between these two forms for $S^{4}$ when $A$ is a discrete $\pi$-quasitriangular multiplier Hopf algebra.

(2) Assume that $A$ is counimodular. We obtain $\delta_{p}^{-1}=\rho_{p^{-1}} u S(u)^{-1} 1_{p}$ for all $p \in G$.

(3) If $G=\{e\}$, then $A$ is a quasitriangular discrete multiplier Hopf algebra. We obtain the formula $\delta^{-1}=u S(u)^{-1}(\iota \otimes\langle\widehat{\delta}, \cdot\rangle)(R)$, see also [D-VD-W, Proposition 2.9].

2.11 Proposition Let $A$ be a counimodular, discrete multiplier Hopf algebra. Suppose that $A$ is $G$-cograded and let $\pi$ denote a crossing of $G$ such that $A$ is $\pi$-ribbon. Let $m$ denote the grouplike multiplier given by $m=\theta u$. Let $\psi$ denote a right integral on 
$A$. For all $p \in G$, let $\rho_{p}$ denote the scalar crossing scaling. The linear functional $\psi(m \cdot)$ is a $\pi$-trace on $A$ if, for all $p \in G$, we have $\rho_{p}=1$.

Proof. Combine the results of Theorem 2.6 and Corollary 2.10(2).

2.13 Remark Let $A$ be a discrete multiplier Hopf algebra as in Proposition 2.11 and suppose that each component $A_{p}$ is a unital and finite-dimensional algebra. $A$ can be considered as a finite-type Hopf group-coalgebra, in the sense of [T] and [Vir]. The result of Proposition 2.11 can be put in the setting of [Vir, Lemma 7.1].

\section{Acknowledgment}

The author would like to thank A. Van Daele for the many helpful discussions during the preparation of this paper.

\section{Appendix-Examples}

We first prove the existence of infinite-dimensional counimodular, discrete multiplier Hopf algebras which are ribbon. Remark that these multiplier Hopf algebras can not be classical Hopf algebras. Indeed, a Hopf algebra with both integrals and cointegrals has to be finite-dimensional, see [VD, Proposition 5.5]. As mentioned before discrete multiplier Hopf algebras are duals (in the sense of [VD]) of co-Frobenius Hopf algebras. Recall that co-Frobenius Hopf algebras are identified as Hopf algebras with integrals. Let $A$ be a coFrobenius Hopf algebra with a left integral $\varphi$. Let $\widehat{A}$ denote the dual discrete multiplier Hopf algebra, given by $\widehat{A}=\{\varphi(a \cdot) \mid a \in A\}$. The evaluation of $\widehat{A}$ on $A$ is denoted by $\langle\widehat{A}, A\rangle$. When we are dealing with infinite-dimensional algebras, the dual bases should be replaced by the canonical multiplier $W=\sum \widehat{\varphi}\left(\cdot \varphi_{(2)}\right) \otimes S^{-1}\left(\varphi_{(1)}\right)$ in $M(A \otimes \widehat{A})$, see [Z1, Lemma 9]. In the lemma below, the multiplier $W$ is denoted by the formal summation $W=\sum u_{i} \otimes v_{i}$ in $M(A \otimes \widehat{A})$. Observe that, for all $f \in \widehat{A}$, we have that both $W(1 \otimes f)$ and $(1 \otimes f) W$ are elements in $A \otimes \widehat{A}$. 
A.1 Lemma Let $A$ be a co-Frobenius Hopf algebra and suppose that $A$ is coquasitriangular (or cobraided) in the sense of [Ka, Definition VIII.5.1]. Then the dual $\widehat{A}$ is a discrete quasitriangular multiplier Hopf algebra.

Proof. As explained above, $\widehat{A}$ is a multiplier Hopf algebra of discrete type. Let $r$ denote a linear form on $A \otimes A$, making $A$ into a coquasitriangular Hopf algebra. Let $W=\sum u_{i} \otimes v_{i}$ denote the formal summation of the canonical multiplier $W$ in $M(A \otimes \widehat{A})$. We define the multiplier $R$ in $M(\widehat{A} \otimes \widehat{A})$ by

$$
R(f \otimes g)=\sum r\left(u_{i} \otimes u_{j}\right) v_{i} f \otimes v_{j} g \text { and }(f \otimes g) R=\sum r\left(u_{i} \otimes u_{j}\right) f v_{i} \otimes g v_{j}
$$

for all $f, g \in \widehat{A}$.

Observe that both $R(f \otimes g)$ and $(f \otimes g) R$ are elements in $\widehat{A} \otimes \widehat{A}$ because, for all $f \in \widehat{A}$, we have that both $W(1 \otimes f)$ and $(1 \otimes f) W$ are in $A \otimes \widehat{A}$. Further, we use that the product in $\widehat{A}$ is associative. We extend the pairing $\langle\widehat{A} \otimes \widehat{A}, A \otimes A\rangle$ to $\langle M(\widehat{A} \otimes \widehat{A}), A \otimes A\rangle$ in a natural way. Now we easily see that $\langle R, x \otimes y\rangle=r(x \otimes y)$ for all $x, y \in A$.

By [Z1, Lemma 9] and the conditions on $r$, we can prove that $R$ is a generalized $R$-matrix for the multiplier Hopf algebra $\widehat{A}$, in the sense of [D-VD-W, Definition 2.3].

Recall that a coquasitriangular Hopf algebra $A$ is called coribbon if there is a central, invertible functional $\zeta$ in the linear dual $A^{\prime}$ such that

$$
\zeta \circ \mu=\sigma(r) * r *(\zeta \otimes \zeta), \quad \zeta(1)=1 \text { and } \zeta \circ S=\zeta
$$

In these formulas, $\mu$ denotes the product in $A, *$ is the convolution operation on linear forms, $r$ is the universal $R$-form on $A \otimes A, \sigma$ is the fly map on $A \otimes A$ and $S$ is the antipode on $A$. Remark that the functional $\zeta$ is the inverse of the functional used in [Ka, Definition XIV.7(5)]. 
A.2 Lemma Let $A$ be a co-Frobenius Hopf algebra which is coquasitriangular with the universal $R$-form $r$. Suppose that $A$ is coribbon and let $\zeta$ denote the linear functional as before. Then $\zeta$ is a ribbon multiplier in $M(\widehat{A})$, in the sense of Remark 2.3(4).

Proof. As $\widehat{A}$ is a discrete multiplier Hopf algebra, we can apply [VD-Z, Proposition 2.9]. We obtain that $M(\widehat{A})=A^{\prime}$ and so the linear functional $\zeta$ is a central multiplier of $M(\widehat{A})$. Moreover, the multiplier $\zeta$ satisfies

$$
\Delta(\zeta)=\sigma(R) R(\zeta \otimes \zeta), \quad \epsilon(\zeta)=1 \text { and } S(\zeta)=\zeta .
$$

A.3 Example Assume that $0 \neq q \in \mathbb{C}$ and that $q$ is not a root of 1 . Let $A=\mathcal{O}_{q}\left(S L_{2}\right)$ be the quantized function algebra on $S L_{2}$. It is known that $A$ is a coFrobenius Hopf algebra which is unimodular integral, see [M-M-N-N-S-U]. Furthermore, $A$ is coquasitriangular and coribbon, see [L-T, Section 5] and [Ka, Sections VIII.7-XIV.7(6)]. By applying the foregoing lemmas, we know that the dual multiplier Hopf algebra $\widehat{A}$, denoted as $D_{q}\left(s l_{2}\right)$, is a discrete multiplier Hopf algebra which is counimodular, quasitriangular and ribbon. As $D_{q}\left(s l_{2}\right)$ is of discrete type, we have that $M\left(D_{q}\left(s l_{2}\right)\right)=A^{\prime}$, see [VD-Z, Proposition 2.9]. We obtain that $U_{q}\left(s l_{2}\right)$ is a subalgebra of $M\left(D_{q}\left(s l_{2}\right)\right)$ because it is well-known that $U_{q}\left(s l_{2}\right)$ is paired with $\mathcal{O}_{q}\left(S L_{2}\right)$. In [Z2], the algebra structure of $D_{q}\left(s l_{2}\right)$ is described in terms of idempotents and multiplier generators (in $U_{q}\left(s l_{2}\right)$ ).

Let $m$ denote the grouplike multiplier in $M\left(D_{q}\left(s l_{2}\right)\right)$, determined from the ribbon setting. Let $\psi$ be a right integral on $D_{q}\left(s l_{2}\right)$. By Proposition 2.11, we have that $\psi(m \cdot)$ is a trace on $D_{q}\left(s l_{2}\right)$ which is invariant for the antipode on $D_{q}\left(s l_{2}\right)$. In the expression $m=u \theta$, the multiplier $\theta$ is known, see [Ka, XIV.7(6)]. However, to calculate the Drinfel'd multiplier $u$, we need the multiplier $R$ in $M\left(D_{q}\left(s l_{2}\right) \otimes D_{q}\left(s l_{2}\right)\right)$. This calculation is a part of the paper [Z2]. The approach above is of pure algebraic nature. In literature we only could find infinite-dimensional Hopf algebras which are "topological" quasitriangular. E.g. for the Hopf algebra $U_{q}\left(s l_{2}\right)$, the $R$-matrix is considered in an appropriate completion of $U_{q}\left(s l_{2}\right) \otimes U_{q}\left(s l_{2}\right)$. 
A.4 Example Let $G$ denote an arbitrary group. We give an example of a $G$ cograded $\pi$-quasitriangular multiplier Hopf algebra. Let $\langle A, B\rangle$ be a pairing of two multiplier Hopf algebras. Assume that $B$ is $G$-cograded and let $\pi$ denote a crossing of $G$ on $B$. In $[\mathrm{D}-\mathrm{VD}]$ we have constructed the Drinfel'd double $D^{\pi}$ in this framework. The product and the coproduct depend both on the pairing and on the crossing $\pi$. We have that $D^{\pi}$ is again a $G$-cograded multiplier Hopf algebra and there is a natural crossing of $G$ on $D^{\pi}$, see [D-VD, Proposition 3.13]. Suppose that the pairing $\langle A, B\rangle$ is provided with a cannonical multiplier $W$ in $M(B \otimes A)$. E.g. let $B$ be a Co-Frobenius Hopf algebra and put $A=\widehat{B}$, then $W=\sum \widehat{\varphi}\left(\cdot \varphi_{(2)}\right) \otimes S^{-1}\left(\varphi_{(1)}\right)$ in $M(B \otimes \widehat{B})$ for a left integral $\varphi$ on $B$ and a left integral $\widehat{\varphi}$ on $\widehat{B}$. The embedding of $W$ in $M\left(D^{\pi} \otimes D^{\pi}\right)$ makes $D^{\pi}$ into a $\pi$-quasitriangular multiplier Hopf algebra, see [D-VD-W, Theorem 2.13]. The "finite-type" version of this construction is done in $[\mathrm{Zu}]$.

\section{References}

[A-D-VD] A.T. Abd El-hafez, L. Delvaux and A. Van Daele, Group-cograded multiplier Hopf(*-)algebras, Algebras and Representation Theory 10 (1)(2007), 77-95.

[D-VD] L. Delvaux and A. Van Daele, The Drinfel'd double for group-cograded multiplier Hopf algebras Algebras and Representation Theory 10(3), 197-221.

[D-VD-W] L. Delvaux, A. Van Daele and S.H. Wang, Quasitriangular (G-cograded) multiplier Hopf algebras. J. Algebra 289 (2005), 484-514.

[H] M.A. Hennings, Invariants of links and 3-manifolds obtained from Hopf algebras, J. London Math. Soc 54 (2) (1996), 594-624.

[Ka] C. Kassel, Quantum Groups, Springer, Berlin, 1995. 
[K-R] Louis H. Kauffman and David E. Radford, A necessary and sufficient condition for a finite-dimensional Drinfel'd double to be a ribbon Hopf algebra, J. Algebra 159 (1993), 98-114.

[L-T] R. G. Larson and J. Towber, Two dual classes of bialgebras related to the concepts of "quantum group" and "quantum Lie algebra", Comm. Algebra 19 (1991), 3295-3345.

[M-M-N-N-S-U] T. Masuda, K. Mimachi, Y. Nagakami, M. Noumi, Y. Saburi and K. Ueno, Unitary Representations of the Quantum Groups $S U_{q}(1,1)$ : Structure of the dual space of $U_{q}\left(s_{2}\right)$, Lett. Math. Phys. 19 (1990), 187-194.

[R1] D.E. Radford, The order of the antipode of a finite dimensional Hopf algebra is finite, Amer. J. Math. 98 (1976), 333-355.

[R2] D.E. Radford, On Kauffman's knot invariants arising from finite-dimensional Hopf algebras, in: J. Bergen, S. Montgomery (Eds.), Advances in Hopf algebras, Lecture Notes in Pure and Appl. Math., 158(1994), 205-266.

[T] V.G. Turaev, Homotopy field theory in dimension 3 and crossed groupcategories, Preprint GT/0005291.

[VD] A. Van Daele, An algebraic framework for group duality, Advances in Mathematics 140 (1998), 323-366.

[VD-Z] A. Van Daele and Y. Zhang, Multiplier Hopf algebras of discrete type, J. Algebra 214 (1999), 400-417.

[Vir] A. Virelizier, Hopf group-coalgebras, J. Pure and Applied Algebra 171 (2002), $75-122$.

[Z1] Y. H. Zhang, The quantum double of a co-Frobenius Hopf algebra, Comm. Algebra 27(1999), 1413-1427. 
[Z2] Y. H. Zhang, The discrete quantum group $D q\left(s l_{2}\right)$, Private communication.

[Zu] M. Zunino, Double construction for crossed Hopf coalgebras, J. Algebra, 278 (2004), 43-75. 